\title{
EL TEATRO Y SU INCIDENCIA EN EL DESARROLLO DE LAS HABILIDADES LINGUISTICAS
}

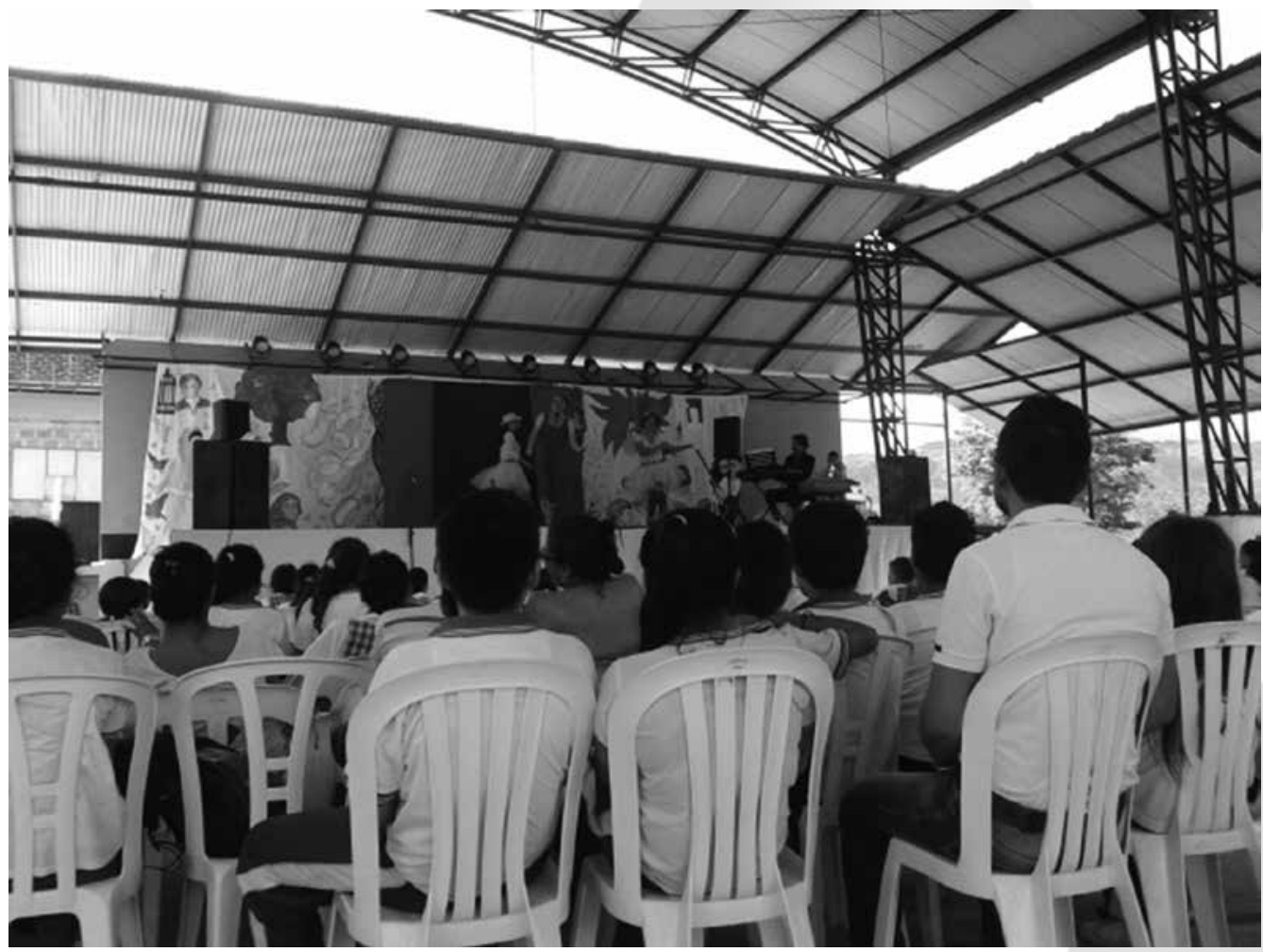

XV Festival de Teatro "La Nariz contra el piso" año 2014, organizado por estudiantes del grado Undécimo de la Institución Educativa María Auxiliadora de Elías, Huila; bajo la coordinación de la profesora Luisa Fernanda Puentes Paredes

Luisa Fernanda Puentes Paredes * Licenciada en Lengua Castellana Lufepupa_7@hotmail.com

\section{RESUMEN}

En el área de Lengua Castellana de la Institución Educativa María Auxiliadora del Municipio de Elías, Huila, así como en Colombia y en el ámbito internacional, se identificó la utilización de prácticas didácticas-pedagógicas muy tradicionales, con la consecuencia de apropiación de estrategias que no siempre posibilitan el desarrollo de habilidades lingüísticas comunicativas de los estudiantes. Por lo anterior, el propósito de la presente investigación fue "Indagar el desarrollo de habilidades lingüísticas comunicativas de los estudiantes de la Institución Educativa María Auxiliadora del municipio de Elías, Huila, mediante la práctica del teatro como estrategia didáctica-pedagógica".

Palabras Clave: TEATRO, FESTIVAL DE TEATRO "LA NARIZ CONTRA EL PISO", HABILIDADES LINGÜÍSTICAS-COMUNICATIVAS, PRÁCTICAS PEDAGÓGICAS.

* Magíster en Educación Universidad Sulcolombiana

1 Proyecto de investigación "El teatro y su incidencia en el desarrollo de las habilidades lingüísticas en los estudiantes de la Institución Educativa María Auxiliadora del Municipio de Elías" realizado durante el año 2014.

2 Licenciada en Educación Básica con énfasis en Humanidades y Lengua Castellana de la Universidad Surcolombiana, aspirante a Magister en Educación con énfasis en Diseño, Gestión y Evaluación Curricular de la Universidad Surcolombiana, Docente de la Institución Educativa María Auxiliadora del Municipio de Elías, Huila. 


\section{ABSTRACT}

In the area of Spanish Language of School Mary Help of the Municipality of Elías, and Huila in Colombia and internationally, using very traditional didactic-pedagogic practices were identified, with the result that no appropriation strategies always enables the development of communicative language skills of students. Therefore, the purpose of this investigation was "Investigate the development of communicative language skills of students of School Mary Help of the municipality of Elias, Huila, through the practice of theater as didactic-pedagogic strategy."

Key words: Theater, Theater Festival "The nose on the floor," Communicative Language Skills, Pedagogical Practices.

\section{A MANERA DE INTRODUCCIÓN}

El propósito de este trabajo de investigación es indagar el desarrollo de las habilidades lingüísticas comunicativas de los estudiantes de la Institución Educativa María Auxiliadora del municipio de Elías, Huila. Para lograrlo, el proceso investigativo se argumenta sobre tres ejes fundamentales:

a) Teórico: Por la importancia que tiene el desarrollo de las habilidades lingüísticas comunicativas en los estudiantes, según los lineamientos curriculares de Lengua Castellana del Ministerio de Educación Nacional.

b) Práctico: Por la pertinencia curricular y por la pertinencia social que tiene el desarrollo de habilidades lingüísticas comunicativas en el proceso de formación personal, comunitario y social de los estudiantes.

c) Social: Por la contribución al desarrollo de las habilidades lingüísticas comunicativas del teatro como estrategia didáctico-pedagógica.

Con estos tres ejes, mediante la apropiación de las artes que han hecho los alumnos y docentes de la Institución Educativa María Auxiliadora, la comunidad educativa ha diseñado, ejecutado y conservado el "FESTIVAL DE TEATRO: LA NARIZ CONTRA EL PISO" como una estrategia pedagógica que surgió en el aula hace catorce años, en 1999. Esta iniciativa germinó a partir de la necesidad de potenciar en los educandos su habilidad para leer de manera comprensiva un texto, para saber escuchar, y consecuentemente, para saber escribir y hablar ante un público de maneras lúdica, pedagógica y creativa.

\section{REFERENTES TEÓRICOS}

Dado el énfasis cualitativo del estudio investigativo, las categorías teóricas que sustentan el proceso investigativo desde el punto de vista teórico, han sido planteamientos de pedagogos, sicólogos, lingüistas, académicos, gobernantes, filósofos, provenientes de equipos de investigadores, asesores del Ministerio de Educación, entre otros, que han postulado el problema en términos concretos para plantear soluciones, enfoques, modelos y estrategias, que expliquen sistemáticamente y con base en estadísticas y análisis por qué el grueso de la población escolar y hasta universitaria no lee, o si lee, no comprende el significado global del texto.

A pesar de la asimilación de los modelos teóricos propuestos por los autores mencionados y su relación con el ámbito escolar, el problema subsiste y se incrementa con el auge de la lectura rápida y fragmentada en los soportes tecnológicos. Los numerosos diagnósticos y modelos teórico-prácticos exponen claramente estadísticas abrumadoras de lo que sucede en Instituciones educativas, universitarias y técnicas.

Para sustentar teóricamente la praxis investigativa, se hizo una indagación de los 
planteamientos teóricos del concepto TEATRO y de la incidencia de la práctica del teatro en el aula escolar para desarrollar habilidades lingüísticas comunicativas en procesos como leer, escribir, escuchar y hablar de los estudiantes de la Institución Educativa María Auxiliadora del municipio de Elías, Huila.

Ahora bien, definir conceptualmente las habilidades lingüísticas desde una perspectiva curricular es comprender que procesos como leer, escribir, hablar y escuchar, constituyen el eje por el que se construye el significado en actos reales de comunicación. Así por ejemplo, "para la tradición lingüística y algunas teorías psicológicas, se considera el acto de "leer" como comprensión del significado del texto. Algo así como el reconocimiento de un sujeto lector que se basa en el reconocimiento y manejo de un código (la lengua,) y que tiende a la comprensión" .

Sin embargo, cuando se habla del significado del texto, se ha de tener en cuenta tres factores para su comprensión: el reconocimiento del sentido global del texto, la intención comunicativa del autor, y los contextos socioculturales del lector. Comprender el significado global del texto es identificar la secuencia temática y su respectiva coherencia, es, en últimas, reconocer su macroestructura. A su vez la intención comunicativa tiene un propósito particular de comunicación, que se evidencia en la concreción del acto de habla propio de la función pragmática de los discursos. Y definitivamente el conocimiento del contexto, valida la tesis según la cual a cada época, a cada lugar y a cada situación comunicativa le corresponde una noción espacio-temporal particular.

De otro lado, ESCRIBIR, según los lineamientos de lengua castellana del Ministerio de Educación Nacional es, "un proceso que a la vez es social e individual en el que se configura un mundo y se ponen en juego saberes, competencias, intereses, y que a la vez está determinado por un contexto socio-cultural y pragmático que determina el acto de escribir: escribir es producir el mundo" .

Así, en el texto de lineamientos curriculares ya citado se lee:

Respecto a los actos de "escuchar" y "hablar", es necesario comprenderlos de manera similar. Es decir, en función de la significación y la producción del sentido. Escuchar, por ejemplo, tiene que ver con elementos pragmáticos como el reconocimiento de la intención del hablante, el reconocimiento del contexto social, cultural, ideológico desde el cual se habla; además está asociado a complejos procesos cognitivos ya que, a diferencia del acto de leer en el que se cuenta con el impreso como soporte de la significación, escuchar implica ir tejiendo el significado de manera inmediata, con pocas posibilidades de volver atrás en el proceso interpretativo de los significados. A su vez, hablar resulta ser un proceso igualmente complejo, es necesario elegir una posición de enunciación pertinente a la intención que se persigue, es necesario reconocer quién es el interlocutor para seleccionar un registro de lenguaje y un léxico determinado, etcétera .

La propuesta didáctica-pedagógica y curricular del Ministerio de Educación Nacional en el área de lengua castellana, configura el marco general de los referentes de formación en lenguaje de los procesos comunicativos y de significación. Es así como el sentido y las funciones de la pedagogía y del currículo educativo atienden las necesidades, los intereses y los procesos de los principales actores, las personas, los grupos, las etnias, entre otras poblaciones, para el desarrollo de sus potencialidades.

3 Ministerio de Educación Nacional. Lineamientos curriculares de Lengua Castellana. Bogotá. 1998. p. 48 
En este proceso investigativo se resaltaron los conceptos: TEATRO y HABILIDADES LINGÜÍSTICAS-COMUNICATIVAS.

TEATRO: En el Diccionario de la Real Academia de la Lengua Española leemos que la palabra teatro proviene del latín theātrum; etimológicamente, está referido al edificio o sitio destinado a la representación de obras dramáticas o a otros espectáculos públicos propios de la escena. Sin embargo, por teatro se conoce el $<<$ Conjunto de todas las producciones dramáticas de un pueblo, de una época o de un autor $>>$; es el arte de componer obras dramáticas, o de representarlas ante un público.

Con base en la anterior acepción, Rodrigo Durán Rosero define el teatro como una "actividad artística que se representa a través de un o unos actores, que cuenta una fábula, una historia a un público presente y que se agota en el término de su proceso" . El teatro ha sido para la humanidad, la manifestación viva de la realidad. En sus orígenes fue de gran importancia para significar el entorno social, cultural, y ritual de las comunidades primitivas y para establecer nexos comunicativos.

hABILIDADES LINGUístICAS COMUNICATIVAS: Según el Ministerio de Educación Nacional en la Ley General de Educación de 1994, los objetivos generales de la educación básica -Artículo 20- numeral b, contempla: "Desarrollar las habilidades comunicativas para leer, comprender, escribir, escuchar, hablar y expresarse correctamente"; siendo así, asimilamos la habilidad lingüística con carácter de habilidad comunicativa, puesto que se deriva de la competencia o capacidad para interactuar en diversos contextos". A partir de esta directriz estatal, algunos teóricos han definido conceptualmente las habilidades lingüísticas: leer, escribir, escuchar, hablar, así:

- LEER: Etimológicamente leer proviene del latín legĕre que significa "Pasar la vista por lo escrito o impreso comprendiendo la significación de los caracteres empleados", pero también "Comprender el sentido de cualquier otro tipo de representación gráfica". Y así, son múltiples las definiciones que develan el significado del término, pero ¿qué sucede cuando escuchamos una sinfonía de Beethoven, u observamos un cuadro pictórico de algún autor desconocido, o conocido, no son acaso lecturas de representaciones del mundo, creadas por el arte a partir de un referente de la realidad?

La conceptualización teórica del acto "leer" tiene, desde la antigüedad, una definición mecánica e instrumental que hasta hace algunos siglos ha sido superada, por ejemplo: "Unos doscientos años después de haberse creado el alfabeto griego, Platón explicaba el acto de leer del siguiente modo: "distinguir las letras separadas a la vez por el ojo y por el oído en orden que, cuando más tarde se lo escuche hablado o se lo vea escrito, no será confundido por su posición". Según Kenneth Goodman "los lectores interactúan y transactúan a través de los textos. Esto quiere decir que lo que el lector le aporta en términos de conocimientos, valores, experiencias, y creencias es tan importante como la que el autor aporta a la creación del texto" .

Es por esto que el acto de leer -entendido como un fenómeno del lenguaje-, es una práctica social indispensable para el hombre en todas sus realidades, porque solo con su realización podemos concebir el mundo y significarlo. Así, "el arte de leer, de hacer de un texto una creación propia, y el expresarse y manifestarse por escrito, compete a cada individuo y a su personalidad singular y diferente de la de los demás". Siendo así, 
se considera entonces, que el acto de leer implica la relación autor-texto-lector-contexto, ya que en esa interacción se involucran habilidades cognitivas y lingüísticas del lector, sus saberes previos y la construcción dialógica, como lo reafirman Emilia Ferreiro y Ana Teberovsky en su obra Los sistemas de escritura en el desarrollo del niño .

- ESCRIBIR: etimológicamente el término proviene del Latín scribĕre que significa "Representar las palabras o las ideas con letras u otros signos trazados en papel u otra superficie"; si bien es cierto, la escritura es algo que aprendemos desde los primeros años en sentido estricto de la palabra, la escritura es, a su vez, un proceso de pensamiento complejo que implica razonamiento abstracto, conocimiento profundo de la lengua y desarrollo de capacidades para crear, por medio de palabras, nuevos significados.

Se recogen entonces, los aportes de Ana Teberovsky en el capítulo "Construcción de escritura a través de la interacción grupal" : "(...) la escuela ha considerado fundamentalmente a la escritura como una actividad individual, "para si", del sujeto, olvidando que ella es el resultado de un esfuerzo colectivo de la humanidad, cuya función es social". De otro lado, "Algunos estudiosos han considerado que la escritura es un proceso paralelo al del habla, ya que tanto hablar y escuchar, por una parte, cómo escribir y leer por otra, son hechos lingüísticos".

- ESCUCHAR: De acuerdo con el Diccionario de la Real Academia de la Lengua Española, escuchar es, en primer lugar, prestar atención a lo que se oye. Es a su vez dar oídos, atender a un aviso, consejo o sugerencia.

De acuerdo con las indicaciones de los Lineamientos curriculares de Lengua Castellana, el acto de "escuchar" debe ser comprendido en función de la significación y la producción del sentido: "Escuchar, por ejemplo, tiene que ver con elementos pragmáticos como el reconocimiento del contexto social, cultural, ideológico desde el cual se habla" . Lo que significa que el acto de escuchar no se limita a la atención de los discursos orales, sino a la comprensión del sentido y su significado en contextos y actos reales de comunicación.

- HABLAR: Según el Diccionario de la Real Academia de la Lengua Española; hablar es articular, proferir palabras para darse a entender, es pronunciar un discurso u oración, es tratar, convenir, concertar, es expresarse de uno u otro modo, es manifestar, en lo que se dice, cortesía o benevolencia o al contrario, o bien, emitir opiniones favorables o adversas acerca de personas o cosas. Es a su vez, dirigir la palabra a alguien; además, explicarse o darse a entender por medio distinto del de la palabra.

Con esta aproximación conceptual a los términos de referencia TEATRO y HABILIDADES LINGUÍSTICAS-COMUNICATIVAS, en la presente investigación descriptiva se podrá explicar por qué se plantea el Problema "¿De qué manera el teatro incide en el desarrollo de las habilidades lingüísticas comunicativas de los estudiantes de la Institución Educativa María Auxiliadora del Municipio de Elías?"; además, se vislumbran posibles soluciones desde el manejo histórico de lo que ha significado el ejercicio del teatro -como representación de la condición humana-, puesto que no es una actividad ocasional o pasajera, sino que se puede llevar al aula escolar como una forma didáctica-pedagógica que dignifique la relación de la comunidad académica con su entorno. 


\section{METODOLOGÍA APLICADA}

TIPO DE INVESTIGACIÓN: La presente investigación se apoyó en una integración del método cualitativo con el método cuantitativo, lo que resulta inevitable para determinar alcances, enfoques, intereses y tendencias de los sujetos implicados en la población intervenida. No obstante, la investigación es descriptiva de tipo cualitativo-interpretativa, lo que induce un proceso investigativo mixto, siguiendo la idea de Roberto Hernández Sampieri: "La meta de la investigación mixta no es reemplazar a la investigación cuantitativa ni a la investigación cualitativa, sino utilizar las fortalezas de ambos tipos de indagación combinándolas y tratando de minimizar sus debilidades potenciales".

Siendo que el estudio de la población se ocupará de describir las condiciones de las metodologías implementadas en el aula escolar -en este caso, grupos de educación básica y media-, se logrará alcanzar el objetivo general, "indagar el desarrollo de las habilidades lingüísticas comunicativas de los estudiantes de la Institución Educativa María Auxiliadora de Elías, Huila, mediante la práctica del teatro como estrategia didáctica-pedagógica", por medio de la sistematización de los fenómenos observados durante la investigación, puesto que, siguiendo a Hernández Sampieri, "Los estudios descriptivos buscan especificar las propiedades, las características y los perfiles de grupos, comunidades, procesos, objetos o cualquier otro fenómeno que se someta a un análisis" .

PARTICIPANTES: La Población es la comunidad escolar de la Institución Educativa María Auxiliadora y la muestra representativa es de 66 estudiantes de grado sexto a undécimo de la sede principal de Elías, Huila, para un total de once grupos con seis estudiantes de cada grado: tres varones y tres niñas con niveles variados de desempeño en la escala de MUY BAJO, BAJO, BÁSICO ALTO y SUPERIOR durante el primer periodo académico del año 2014.

PROCEDIMIETOS: La metodología de la presente investigación se hizo por procesos; en primer lugar, un rastreo de antecedentes del Festival de Teatro "La Nariz contra el piso", con la entrevista al director del Festival, profesor Luis Ernesto Gómez. Enseguida se aplicó una encuesta a la población seleccionada, para diagnosticar gustos, intereses, motivaciones y dificultades que consideran en torno a habilidades lingüísticas comunicativas. Y finalmente, una exploración teatral que indagó la incidencia de la práctica del teatro en el desarrollo de las habilidades lingüísticas comunicativas, teniendo en cuenta conceptos mínimos del teatro como Actores, Escenario, Técnica Vocal, Expresión Corporal, y Creación Colectiva.

A continuación, se presentan las herramientas UNIDAD DE ANÁLISIS DE LOS ESTUDIANTES, SISTEMATIZACIÓN Y ANÁLISIS DE LA INFORMACIÓN Y TESTIMONIOS DE LOS ESTUDIANTES:

\footnotetext{
9 Citado por Berta Braslavsky, ob., cit., p. 47.

10 Martha Sastrías. Caminos a la Lectura. Diversas propuestas para despertar y mantener la afición por la lectura en los niños. Editorial Pax, México, 2001.

11 Emilia Ferreiro y Ana Teberovsky. Los sistemas de escritura en el desarrollo del niño, México, Siglo XXI Editores, 1995, p 344-351.

12 Emilia Ferreiro y Margarita Gómez Palacio (compiladoras). Nuevas perspectivas sobre los procesos de lectura y escritura, México, Siglo XXI, p. 156.

13 Citado por Berta Braslavsky, ob., cit., p 43.

14 Lineamientos Curriculares, p 50.

15 Roberto Hernández Sampieri y otros. Metodología de la Investigación, Interamericana Editores. México, 2010, p. 544

16 Ibíd., p 80.
} 


\begin{tabular}{|c|c|c|c|c|c|c|c|}
\hline \multicolumn{4}{|c|}{ UNIDAD DE ANÁLISIS DE LOS ESTUDIANTE } & 45. & JULIANA JAIME GARZON & $9 B$ & ALTO \\
\hline NOI & BRE COMPLETO & RADO & DESEMPEÑO & 46. & ADRIANA LUCIA ROJAS & $9 B$ & ALTO \\
\hline 1. & CLAUDIA PEREZ PIAMBA & $6 \mathrm{~A}$ & BAJO & 47. & ALDEMAR RODRIGUEZ & $9 B$ & BÁSICO \\
\hline 2. & ANGIE GONZALEZ & $6 \mathrm{~A}$ & BAJO & 48. & DUVAN CAMARGO & $9 B$ & BAJO \\
\hline 3. & LAURA MARIA AMAYA & $6 \mathrm{~A}$ & SUPERIOR & 49. & ANDRES FELIPE CALERO & $10 \mathrm{~A}$ & ALTO \\
\hline 4. & ERVIN CLAROS & $6 \mathrm{~A}$ & BAJO & 50. & DUVAN FERNEY CASTRO & $10 \mathrm{~A}$ & BÁSICO \\
\hline 5. & JUAN DAVID CAMARGO & $6 \mathrm{~A}$ & BÁSICO & 51. & ANGELICA MARIA AMAYA & $10 \mathrm{~A}$ & ALTO \\
\hline 6. & JAIRO STIVEN CUELLAR & $6 \mathrm{~A}$ & SUPERIOR & 52. & MARIA ISABEL PRIETO & $10 \mathrm{~A}$ & BASICO \\
\hline 7. & YELITZA CHILITO & $6 \mathrm{~B}$ & BAJO & 53. & ANDREA PLAZAS & $10 \mathrm{~A}$ & BÁSICO \\
\hline 8. & ADRIANA GONZALEZ & $6 \mathrm{~B}$ & BÁSICO & 54. & KELLY JESSENIA SALAS & $10 \mathrm{~A}$ & BASICO \\
\hline 9. & TATIANA CARVAJAL & $6 \mathrm{~B}$ & SUPERIOR & 55. & ANYI HERNANDEZ & $10 \mathrm{~B}$ & BÁSICO \\
\hline 10. & SEBASTIAN TANDIOY & $6 \mathrm{~B}$ & BÁSICO & 56. & MARIO LOZANO & $10 \mathrm{~B}$ & BAJO \\
\hline 11. & JORGE TOVAR & $6 \mathrm{~B}$ & ALTO & 57. & JOSÉ IGNACIO LOZADA & $10 \mathrm{~B}$ & BÁSICO \\
\hline 12. & JUAN ANDRÉS TOLEDO & $6 \mathrm{~B}$ & BÁSICO & 58. & CAROL SERRATO & $10 \mathrm{~B}$ & BÁSICO \\
\hline 13. & VICTOR CUELLAR & $7 \mathrm{~A}$ & BÁSICO & 59. & ALEJANDRA PRIETO & $10 \mathrm{~B}$ & ALTO \\
\hline 14. & YULY HERNANDEZ & $7 \mathrm{~A}$ & BÁSICO & 60. & DANIEL TOVAR DIAZ & $10 \mathrm{~B}$ & ALTO \\
\hline 15. & ANA MARÍA MURCIA & $7 \mathrm{~A}$ & SUPERIOR & 61. & KARLA CASTAÑO & 11 & BÁSICO \\
\hline 16. & MAYRA LILIANA LLANOS & $7 \mathrm{~A}$ & SUPERIOR & 62. & LUZ ENITH GOMEZ & 11 & ALTO \\
\hline 17. & JUAN CAMILO MATIZ & $7 \mathrm{~A}$ & BAJO & 63. & YAMIL MENESES & 11 & ALTO \\
\hline 18. & JUAN JOSÉ JIMENEZ & $7 \mathrm{~A}$ & BAJO & 64. & JEIDY YULIANA BERMEO & 11 & BÁSICO \\
\hline 19. & YURY POSSO & $7 \mathrm{~B}$ & BAJO & 65. & NORVEY MUÑOZ & 11 & BÁSICO \\
\hline 20. & MAYERLI ROJAS GOMEZ & $7 \mathrm{~B}$ & BÁSICO & 66. & MAURO BORDA & 11 & BÁSICO \\
\hline 21. & KEIDY ALEJANDRA & $7 \mathrm{~B}$ & ALTO & & & & \\
\hline 22. & CAMILO RAMIREZ & $7 \mathrm{~B}$ & ALTO & & & & \\
\hline 23. & JUAN DIEGO TORRES & $7 \mathrm{~B}$ & BÁSICO & & & & \\
\hline 24. & ESTEBAN CARVAJAL & $7 \mathrm{~B}$ & BÁSICO & & & & \\
\hline 25. & SEBASTIAN MORALES & $8 \mathrm{~A}$ & BÁSICO & & & & \\
\hline 26. & YULEIDY DORADO & $8 \mathrm{~A}$ & BAJO & & & & \\
\hline 27. & YAIR VARGAS SOTELO & $8 \mathrm{~A}$ & BAJO & & & & \\
\hline 28. & LAURA CAMILA SERRATO & $8 \mathrm{~A}$ & ALTO & & & & \\
\hline 29. & DORIS ADRIANA VERU & $8 \mathrm{~A}$ & BÁSICO & & & & \\
\hline 30. & ADRIAN CAMILO CARDOZC & $8 \mathrm{~A}$ & SUPERIOR & & & & \\
\hline 31. & ALEXANDER TOLEDO & $8 \mathrm{~B}$ & BÁSICO & & & & \\
\hline 32. & HENRRY GIRON & $8 \mathrm{~B}$ & BÁSICO & & & & \\
\hline 33. & LEANDRO ARTUNDUAGA & $8 \mathrm{~B}$ & SUPERIOR & & & & \\
\hline 34. & ANDREA VARGAS & $8 \mathrm{~B}$ & BÁSICO & & & & \\
\hline 35. & SILVIA VALDERRAMA & $8 \mathrm{~B}$ & SUPERIOR & & & & \\
\hline 36. & VALENTINA CUENCA & $8 \mathrm{~B}$ & BÁSICO & & & & \\
\hline 37. & LINA MARIA CUELLAR & $9 \mathrm{~A}$ & ALTO & & & & \\
\hline 38. & YURIANA ROJAS & $9 \mathrm{~A}$ & BÁSICO & & & & \\
\hline 39. & JOSÉ DANIEL GONZALEZ & $9 \mathrm{~A}$ & BÁSICO & & & & \\
\hline 40. & MERY CASTAÑO & $9 \mathrm{~A}$ & BÁSICO & & & & \\
\hline 41. & SANTIAGO BERMEO & $9 \mathrm{~A}$ & BAJO & & & & \\
\hline 42. & SEBASTIAN RODRIGUEZ & $9 \mathrm{~A}$ & SUPERIOR & & & & \\
\hline 43. & JAVIER MUÑ̃Z & $9 B$ & ALTO & & & & \\
\hline 44. & LEANDRO SALGADO & $9 \mathrm{~B}$ & BÁSICO & & & & \\
\hline
\end{tabular}


SISTEMATIZACIÓN Y ANÁLISIS DE LA INFORMACIÓN: Interpretación del resultado de la encuesta aplicada a los 66 estudiantes de la Institución Educativa María Auxiliadora:

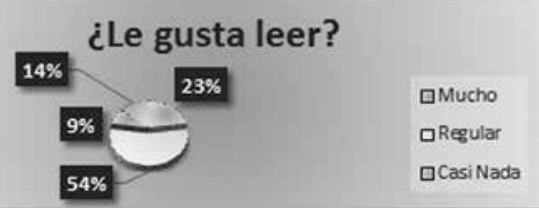

Tabla 1: Unidad de análisis

La gráfica anterior muestra que a los estudiantes de la IE María Auxiliadora les gusta leer de manera regular, representando el $54 \%$ de la población encuestada; mientras al $23 \%$ de los estudiantes les gusta leer mucho; sin embargo, el $14 \%$ de los estudiantes no lee nada por gusto personal, y el $9 \%$ de la población encuestada indica que casi no le gusta leer.

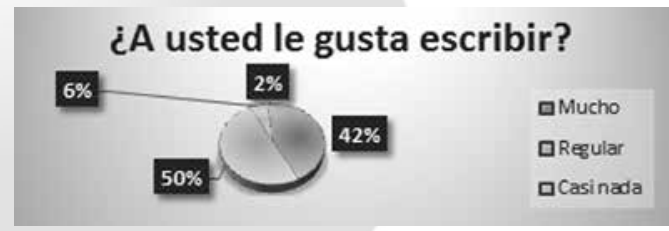

Figura 3: Gráfica circular

Se identifica que el $50 \%$ de la población encuestada le gusta escribir de manera regular, mientras que el $42 \%$ sienten mucho gusto cuando escriben, sin embargo el $6 \%$ de la población respondió que casi nada le gusta escribir y el $2 \%$ absolutamente nada.

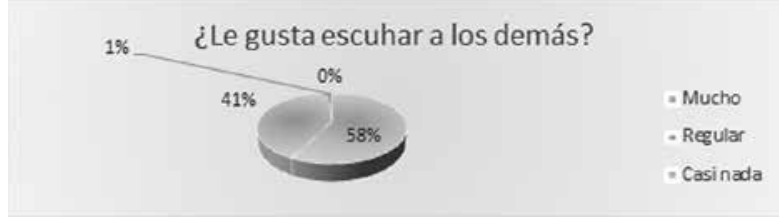

Figura 4: Gráfica circular

De acuerdo con la gráfica anterior al $58 \%$ de la población encuestada le gusta mucho escuchar a los demás, el $41 \%$ le gusta de manera regular y el $1 \%$ escasamente le gusta escuchar.

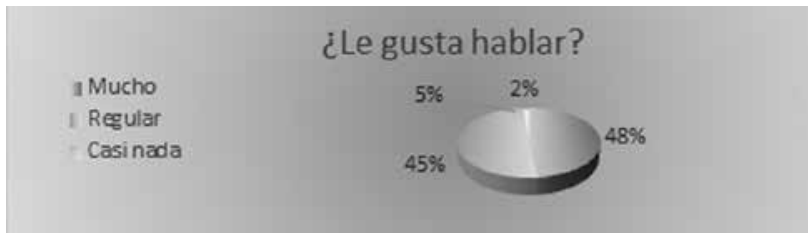

Figura 5: Gráfica circular

De las habilidades lingüísticas comunicativas que más les gustan a los estudiantes encuestados es hablar en un $48 \%$ representado, sin embargo la gráfica anterior nos muestra que el $45 \%$ les gusta de manera regular, el $5 \%$ casi nada y el $2 \%$ no les gusta hablar. 
TESTIMONIO DE LOS ESTUDIANTES: A continuación se registran los testimonios de alguno de los estudiantes tal como se recogieron en el trabajo de campo, luego de realizada la exploración con los talleres de teatro.

Mayra Liliana Llanos: "Fue una experiencia muy útil para mí, ya que me permitió ver la literatura de otra forma, darme cuenta de que leer me hace bien. Aprendí a saber escuchar a los demás y a expresarles mis opiniones, aprendí a entender una lectura y a representarla en un escenario".

Andrés Felipe Calero: "La práctica del teatro ha infundo de alguna manera en mi vida a desarrollar las habilidades lingüísticas e intelectuales. Por ejemplo con los talleres que hacíamos que en cinco minutos teníamos que hacer una historia, nos ayudaba a pensar, a imaginar, y pues, llevar nuestras ideas a cabo. La verdad la lectura mejoró cuando leíamos los capítulos de historias para representarlas en imágenes y escenas. También la forma de dejar la pena y expresar nuestras ideas a los demás sin que la pena nos destruya y no nos deje decir nuestras ideas para que los demás nos entiendan".

Angélica María Amaya: "Nos ha ayudado a quitar nuestros miedos, a tener muchas habilidades, nos ha ayudado a compartir con nuestros amigos, especial en conocer a las demás personas, los niños de los otros salones, y pues, nos ha expresado que por medio de el podemos expresar nuestros sentimientos y emociones".

\section{CONCLUSIONES}

Al establecer el estado actual del proceso de diagnóstico de las habilidades lingüísticas-comunicativas en la Institución Educativa María Auxiliadora del Municipio de Elías, se logró evidenciar que hay diferencias entre el currículo propuesto en el ámbito nacional y en el institucional, con el currículo desarrollado en las clases de Lengua Castellana de la Institución Educativa María Auxiliadora. Según el diagnóstico, el modelo predominante en las clases de Lengua Castellana está centrado en transmisión y recepción de contenidos, con prelación del dominio casi exclusivo del sistema de representación lingüístico natural del profesor.

Con la exploración del teatro en el aula escolar como estrategia didáctica y la forma de entender la clase y los roles de profesores y estudiantes, se evidencia que el modelo de recepción y apropiación del conocimiento y en particular el reconocimiento de las habilidades comunicativas, genera cambio y progreso en el desarrollo de habilidades expresivas, por medio de la práctica teatral; por lo tanto, es un modelo que se puede trabajar desde diversas perspectivas y con la comunidad educativa que ha sido impactada positivamente por un evento cultural que los incluye a todos: padres de familia, estudiantes, profesores, administrativos y que tiene una tradición institucionalizada en la población.

Frente a la pregunta de investigación: ¿De qué manera el teatro incide en el desarrollo de las habilidades lingüísticas de los estudiantes de la Institución Educativa María Auxiliadora del Municipio de Elías? se puede concluir que la exploración teatral desarrollada con los 66 estudiantes de la Institución mostró como resultado mejoramiento en sus habilidades comunicativas, debido a que el trabajo desarrollado durante los talleres de teatro, exigía de parte del estudiante practicar su habilidad para leer, escribir, escuchar y hablar.

Como se pudo observar en el vídeo que se hizo del Festival de Teatro "La nariz contra 
el piso", la práctica teatral sí fue un eje integrador de las esferas del conocimiento, pues mediante juegos sencillos de acondicionamiento físico. Por ejemplo, el estudiante agudizó su habilidad de escucha y de interpretación ya que el acto de escuchar va más allá de la recepción de un acto locutivo, el acto de escuchar implica interpretación del significado del mensaje y finaliza en la realización concreta de este acto de habla. En muchas ocasiones -y casos específicos en los estudiantes-, el acto de escuchar trascendía de la recepción a la interpretación y realización concreta de los discursos comunicados. Es decir, los estudiantes asimilaban con propiedad lo que debían mejorar en su interpretación teatral. De igual forma se concluye, que la habilidad para leer y hablar puede optimizarse mediante la práctica constante del arte teatral, pues la dicotomía actor/público durante la práctica de la lectura en voz alta y de la expresión discursiva, minimiza notablemente factores como miedo, timidez y aislamiento, a la vez que potencia creatividad, seguridad y confianza para desempeñarse con mayor propiedad en un escenario y en las praxis discursivas cotidianas.

Por lo anterior, podría afirmarse claramente que el teatro, como estrategia didáctica-pedagógica, incide positivamente en el desarrollo de habilidades lingüísticas comunicativas de los estudiantes, como se evidenció en los procesos de exploración realizados en el trabajo de campo de la presente investigación Procesos como leer, escribir, escuchar y hablar, son inherentes al proceso de creación y representación de una obra de arte escénica, y a su vez, transforma las prácticas de enseñanza-aprendizaje basadas en la trasmisión de conocimiento y hace que la persona aprenda -con lúdica, actuación, expresión corporal, juego, entre otras maneras- no solo conocimientos estandarizados, sino también, a respetar diferencias, a convivir, a interactuar, a descubrir potencialidades, talentos artísticos y sobre todo, que genere cambios en su manera de pensar y de actuar, por medio de su creatividad.

\section{BIBLIOGRAFIA}

- Braslavsky, Berta. Enseñar a entender lo que se lee. La alfabetización en la familia y en la escuela. Fondo de Cultura Económica. México, 2008. $220 \mathrm{p}$

- Centeno Rojas, Rocío. Habilidades comunicativas 1A / Rocío Centeno Rojas, De yanira Alfonso Sanabria; ilustradores Jaime Pullas [et al.]. Santafé de Bogotá: Libros \& Libres, 1992. 112 p.

- $\quad$ Colectivo El Glayu. Teatro para educar, Ciudad Real: Editora Ñaque, 2006. 133 p.

- Durán, Rosero Rodrigo. El teatro vivo en el Huila a propósito de la ley 1170 . Fondo de Autores Huilenses. Neiva - Huila. 2009.

- $\quad$ Ferreiro, Emilia [et al.]; Nuevas perspectivas sobre los procesos de lectura y escritura, México, Editorial Siglo XXI, 1998, p. 354.

- García, Santiago. Teoría y práctica del teatro, Bogotá, Ediciones La Candelaria, 1989, 276 p.

- González, Fredy Oswaldo. El arte teatral en la escuela, Bogotá: Cooperativa Editorial Magisterio, 2003. 95 p.

- González, Graciela de Díaz Araujo. El teatro en la escuela: estrategias de enseñanza [et al.]; ilustraciones Mario Delhez. Buenos Aires: Aique Grupo Editor, 1998. $160 \mathrm{p}$.

- Gutiérrez, Isabel Cristina [et al.]; Gusto lector un plan para la vida. Prácticas, Maestros y Lectura, Neiva, Editorial Universidad Surcolombiana, 2012, 112 p.

- Herans, Carlos Patiño. Teatro y escuela, México: Distribuciones Fontamara, 2002. $156 \mathrm{p}$.

- Hernández, Sampieri Roberto [et al.]; Metodología de la investigación, México, McGraw Hill, 2010, p. 613. 
- $\quad$ Knébel, María Osipovna. Poética de la pedagogía teatral / María Knébel; traducción de Dalia Mendoza Limón; condensada por Sergio Fernández. México: Siglo Veintiuno Editores, 1991. 180 p.

- Machado, Ana María, 1941. Lectura, escuela y creación literaria, traducción Mario Merlino. Madrid: Anaya, 2002. 133 p.

- $\quad$ Ministerio de Educación Nacional. Estándares Básicos de lenguaje. Bogotá, 2003.

- Ministerio de Educación Nacional. Ley General de Educación, Bogotá, 1994, p. 277.

- Ministerio de Educación Nacional. Lineamientos curriculares de Lengua Castellana, Bogotá, 1998.

- Monroy Calderón, Mario. El teatro como estrategia educativa, teoría y práctica: manual para profesores de educación básica primaria, básica secundaria y media vocacional. Santa Fe de Bogotá: Case, Atlantic Editores, 1995. 180 p.

- Muñoz Hidalgo, Manuel, 1939- El teatro en la escuela para maestros; Fantasía de cartón: ejercicios de teatro en verso para niños y niñas de 4 a 6 años; A Belén, Belén: auto de navidad, Madrid: Editorial Escuela Española, 1994. 130 p.

- $\quad$ Posada de Ceballos, Myriam Ruth [et al.]; Comprensión lectora: un enfoque cognitivo, Neiva, Editorial Universidad Surcolombiana, 2010, 105 p.

- Remacha, Jesús Ángel [et al.]; prólogo Cristina Ma. Ruiz Pérez. Animación a la lectura teatral, Ciudad Real: Naque, 2001. 164 p.

- Rojas Romero, Raúl Alfonso. Propuesta teórica para el diseño de un currículo en la enseñanza del teatro, en las escuelas colombianas, en los grados K-11 / Raúl Alfonso Rojas Romero. México: The University of New México, 1996.

- Wolf, Maryanne. Cómo aprendemos a leer, Historia y Ciencia del cerebro y la lectura. Ediciones B, Barcelona, 2008; 335 p. 